OPEN ACCESS

Edited by:

Jijun Wang,

Shanghai Jiao Tong University, China

Reviewed by:

Lauren Kennedy-Metz,

Harvard Medical School,

United States

Stevan Nikolin,

Black Dog Institute, Australia

*Correspondence:

Tracy A. Dennis-Tiwary

tracy.dennis@hunter.cuny.edu

Specialty section:

This article was submitted to

Clinical Neuroergonomics,

a section of the journal

Frontiers in Neuroergonomics

Received: 11 January 2021

Accepted: 14 April 2021

Published: 19 May 2021

Citation:

Myruski S, Cho H, Bikson M and Dennis-Tiwary TA (2021) Transcranial

Direct Current Stimulation (tDCS)

Augments the Effects of Gamified,

Mobile Attention Bias Modification.

Front. Neuroergon. 2:652162.

doi: 10.3389/fnrgo.2021.652162

\section{Transcranial Direct Current Stimulation (tDCS) Augments the Effects of Gamified, Mobile Attention Bias Modification}

\author{
Sarah Myruski ${ }^{1}$, Hyein Cho $^{2,3}$, Marom Bikson ${ }^{4}$ and Tracy A. Dennis-Tiwary ${ }^{2,3 *}$ \\ ${ }^{1}$ The Pennsylvania State University, State College, PA, United States, ${ }^{2}$ Hunter College, The City University of New York, New \\ York, NY, United States, ${ }^{3}$ The Graduate Center, The City University of New York, New York, NY, United States, ${ }^{4}$ City College, \\ The City University of New York, New York, NY, United States
}

Anxiety-related attention bias $(A B)$ is the preferential processing of threat observed in clinical and sub-clinical anxiety. Attention bias modification training (ABMT) is a computerized cognitive training technique designed to systematically direct attention away from threat and ameliorate $A B$, but mixed and null findings have highlighted gaps in our understanding of mechanisms underlying ABMT and how to design the most effective delivery systems. One neuromodulation technique, transcranial direct current stimulation (tDCS) across the pre-frontal cortex (PFC) may augment the effects of ABMT by strengthening top-down cognitive control processes, but the evidence base is limited and has not been generalized to current approaches in digital therapeutics, such as mobile applications. The present study was a single-blind randomized sham-controlled design. We tested whether tDCS across the PFC, vs. sham stimulation, effectively augments the beneficial effects of a gamified ABMT mobile app. Thirty-eight adults ( $M_{\text {age }}=23.92, S D=4.75 ; 18$ females $)$ evidencing low-to-moderate anxiety symptoms were randomly assigned to active or sham tDCS for 30-min while receiving ABMT via a mobile app. Participants reported on potential moderators of ABMT, including life stress and trait anxiety. ECG was recorded during a subsequent stressor to generate respiratory sinus arrhythmia (RSA) suppression as a metric of stress resilience. ABMT delivered via the app combined with tDCS (compared to sham) reduced AB and boosted stress resilience measured via RSA suppression, particularly for those reporting low life stress. Our results integrating tDCS with ABMT provide insight into the mechanisms of $A B$ modulation and support ongoing evaluations of enhanced ABMT reliability and effectiveness via tDCS.

Keywords: transcranial direct current stimulation, attention bias modification training, mobile application, stress, respiratory sinus arrhythmia

\section{INTRODUCTION}

Anxiety disorders are among the most common and costly of mental health conditions in the United States (Kessler et al., 2005), but only a fraction of patients seek treatment, and a third of those do not respond to current treatment options (Bystritsky, 2006). Thus, recent research and clinical efforts have focused on identifying new targets of intervention and reducing barriers to accessing treatment (e.g., Barak et al., 2008; Rotheram-Borus et al., 2012; Kazdin and Rabbitt, 2013; Dennis and O'Toole, 2014). 
A large body of evidence suggests that individuals evidencing elevated anxiety also show an attention bias $(\mathrm{AB})$ for threatrelevant stimuli, or selective and exaggerated attention to and difficulty disengaging from threat-relevant stimuli (Bradley et al., 2000; Bar-Haim et al., 2007). Techniques such as computerized attention bias modification training (ABMT) have been developed both to examine the causal nature of $\mathrm{AB}$ (e.g., Mathews and MacLeod, 2002) and to serve as a computerized therapy-delivery system. Substantial evidence suggests that training anxious individuals to attend away from threat and toward non-threatening stimuli via ABMT reduces $\mathrm{AB}$ and anxiety severity in clinical and sub-clinical anxiety (e.g., Hakamata et al., 2010) and across ages including children (e.g., Eldar et al., 2008; Pergamin-Hight et al., 2016), although recent meta-analyses (Fodor et al., 2020) also document small effect sizes and raise questions about clinical relevance (e.g., Cristea et al., 2015). Thus, although more evidence is needed, ABMT, which is brief, accessible, cost-effective, and low-toxicity, is a promising new anxiety- and stress-reduction intervention for people experiencing both clinical and sub-clinical anxiety, and for whom intensive treatments may be too time-consuming and cost-prohibitive.

In addition, there remain significant gaps in our understanding of mechanisms that underlie ABMT, which limits the refinement of efficacious, personalized ABMT techniques. Recent neuroimaging and neurophysiological studies point to the potential mechanistic role of pre-frontal-cortex (PFC)-mediated changes in attention control (Cisler and Koster, 2010; Heeren et al., 2013; Dennis-Tiwary et al., 2016). Several models posit that anxiety-related $\mathrm{AB}$ may result from disruptions in the ability to recruit top-down attention control (e.g., MacLeod et al., 1986; Bishop et al., 2004; Cisler and Koster, 2010; Dennis-Tiwary et al., 2019), which would be signaled by reduced activation of the PFC, in particular the dorsal lateral PFC (DLPFC; Bishop, 2009; Browning et al., 2010).

Prior studies (e.g., den Uyl et al., 2018) have directly tested this causal hypothesis by manipulating recruitment of the PFC during ABMT procedures using transcranial direct current stimulation (tDCS). $\mathrm{tDCS}$ is a portable battery-powered device that delivers low-intensity $(\sim 2 \mathrm{~mA})$ direct electric current through electrodes positioned over the scalp (Bikson et al., 2016; Woods et al., 2016). When this current reaches the neural tissue, the polarization of the resting membrane potential is shifted (Radman et al., 2009) modulating ongoing plasticity (Fritsch et al., 2010; Kronberg et al., 2017, 2020), for example plasticity produced by ABMT (Heeren et al., 2015). tDCS can be used both in academic and clinical centers and, when appropriate design steps are taken, at remote settings (e.g., work, home; Charvet et al., 2015). A sham-controlled approach allows comparison of active tDCS stimulation throughout ABMT, vs. a brief ramp up and ramp down at the start and end of the ABMT session. Sham has been shown to be indistinguishable from active tDCS from the participants' perspective (Gandiga et al., 2006), yet does not impact brain function (Nitsche et al., 2008). Thus, combining this approach with a cognitive intervention, like ABMT delivered to both active tDCS and sham groups, provides insight into the neural mechanisms underlying training efficacy.
Two recent studies provide evidence that tDCS nominally targeting the left DLPFC influences ABMT efficacy (Clarke et al., 2014; Heeren et al., 2015). For example, Clarke et al. (2014) showed that, among a non-anxious sample, those who received tDCS vs. sham (placebo) while completing ABMT showed changes in $\mathrm{AB}$ in either targeted direction - both toward and away from threat. This suggests that tDCS enhances AB plasticity, however directed. A subsequent study (Heeren et al., 2015) selecting for high trait anxiety adults (who did not show $\mathrm{AB}$ at baseline), found that combining ABMT and $\mathrm{tDCS}$ reduced an eye tracking index of $\mathrm{AB}$ (duration of gaze fixated on threat). However, another recent study focused on combining ABMT and tDCS among participants with alcohol dependence (den Uyl et al., 2018), no main effects or interactions on $A B$ were found. Taken together, these studies document that the impact of tDCS on ABMT is mixed. However, particularly regarding anxiety, existing research suggest that tDCS augments the impact of $\mathrm{ABMT}$ on $\mathrm{AB}$ as a target cognitive process in the etiology and maintenance of anxiety but may interact with ABMT in an individual- and protocol-specific manner.

While computerized ABMT techniques significantly reduce treatment barriers, they are typically administered on a desktop computer in a laboratory or clinic setting, which remain difficult to access. In addition, traditional ABMT techniques are often described as repetitive and engagement and motivation by participants tends to be low (Dennis and O'Toole, 2014). To address these barriers, we have created a mobile version of ABMT that is more engaging than the traditional ABMT protocol and can be used on mobile devices. This commercially available mobile application or "app" (for iOS devices like iPhones), called Personal Zen, takes the core components of the gold-standard ABMT protocol and puts them in the context of an appealing game (see Dennis and O'Toole, 2014). It further incorporates video game-like features such as animated characters and sound effects. Like traditional ABMT, attention is still systematically redirected away from threat-relevant stimuli (angry faces).

We have recently demonstrated in two placebo-controlled studies with college students evidencing elevated trait anxiety that this user-friendly and engaging version of ABMT reduced anxiety, stress reactivity, and $\mathrm{AB}$ in a single, lab-based session (Dennis and O’Toole, 2014; Dennis-Tiwary et al., 2016). Moreover, in a placebo-controlled trial including pregnant women who used the app for 30-40 min a week for 1 month, stress reactivity measured via salivary cortisol and subjective anxiety were significantly reduced (Dennis-Tiwary et al., 2017). These data demonstrate that the app is an effective delivery system for ABMT.

Further, since the app has been shown to reduce anxiety in non-clinical samples, the next steps in this line of research are to combine tDCS with app-based ABMT, and explore individual differences contributing to ABMT efficacy. Prior ABMT studies (Pergamin-Hight et al., 2016; Egan and DennisTiwary, 2018; Price et al., 2018) and studies utilizing Personal Zen as the delivery system for ABMT (Dennis-Tiwary et al., 2016, 2017) have further documented moderators of $A B$ plasticity, including anxiety severity and life stress (Wald et al., 2017; Egan and Dennis-Tiwary, 2018), suggesting that in order to 
fully understand mechanisms in ABMT, such moderators should be explored.

The present study uses a single-blind, sham-controlled design to test whether tDCS administered during a gamified, mobile version of ABMT serves to boost beneficial effects. While prior studies have examined gamified ABMT (Dennis and O'Toole, 2014; Dennis-Tiwary et al., 2016) and tDCS (Clarke et al., 2014; Heeren et al., 2015) approaches separately, we build this literature by combining these techniques to test whether ABMT enhancement via tDCS generalizes to current approaches in digital therapeutics. We will test the hypothesis that participants receiving tDCS across the PFC, compared to sham tDCS, will show augmented benefits of ABMT, measured via reduced anxious mood, reduced $\mathrm{AB}$, and enhanced stress resilience [measured via respiratory sinus arrhythmia (RSA) measured during a stressor]. Individual differences in anxiety severity and life stress will be explored as potential moderators of effects.

\section{METHODS}

\section{Participants}

Adults were screened to participate from a pool of undergraduate students and community members at an urban college campus. Participants were excluded if they reported history of epilepsy, irremovable metallic pieces in or around the head, head or neck tattoos, severe skin sensitivity or condition (e.g., eczema) affecting the face or scalp, latex allergies, history of head or traumatic brain injury, use of hearing aid devices, or pregnancy. An a priori power analysis (conducted via $\mathrm{G}^{*}$ Power) showed that a sample size of 42 participants would be sufficient to detect medium effect sizes ( $f=0.31$ and above) across the two target within-subjects measures (pre-tDCS and post-tDCS) at $96 \%$ power. Total of 60 participants were recruited to account for potential data loss. Out of these individuals, seven participants were excluded at phone screen due to one or more of these criteria, 14 chose not to participate following the phone screen, and one participant was excluded before consenting due to presence of a fixed metal retainer in the mouth (see Figure 1 for a participant selection flowchart). The final sample of 38 $\left[M_{\text {age }}=23.92, S D=4.75 ; 18\right.$ (47.4\%) females $]$ reported normal to moderate levels of anxiety according to self-report on the Depression, Anxiety, and Stress Scale (DASS-21; Henry and Crawford, 2005); $M=3.16, S D=3.07$, $M i n=0.00, \operatorname{Max}=$ 13.00). Sample demographics are presented in Table 1. Following informed consent, participants were randomly assigned to one of the two study groups: either active experimental group $(n=18)$ that receives active electrical stimulation or sham control group $(n=20)$ in which there is no stimulation. Prior to data collection, research personnel used an online random sequence generator to determine assignments, which alternated randomly between the two conditions (active tDCS, sham). The sequence was recorded in a log accessible by the research personnel administering the study procedures. For each participant, the assigned condition was implemented by setting a switch on the stimulator box to either "sham on" or "sham off," which was done by the researcher out of view of the participant. Three participants were excluded from ECG analyses due to unusable recordings. The significance of findings did not change when these individuals were removed from other analyses.

This study was approved by the Institutional Review Board (IRB) of Hunter College, CUNY (Protocol 334490) and registered on clinicaltrials.gov (Identifier NCT04348812). The clinical trial protocol approved by the IRB.

\section{Materials and Procedure}

ECG was recorded throughout the entire session. Participants first completed an $\mathrm{AB}$ assessment (the dot probe task) followed by self-report questionnaires. Next, tDCS was applied and administered for $30 \mathrm{~min}$ which included $25 \mathrm{~min}$ of ABMT and a 5-min break. Participants then completed an ECG baseline and $\mathrm{AB}$ assessment, followed by a stressful anagrams task. Finally, a third $A B$ assessment concluded the study session. Self-reported state anxiety and mood was assessed before and after the tDCS administration and the anagrams task. In total, each study session took $\sim 2.5-3 \mathrm{~h}$ to complete.

\section{Transcranial Direct Current Stimulation (tDCS)}

tDCS was administered using a Soterix 1X1 tDCS Limited Total Energy (LTE) Stimulator while participants completed the ABMT app. Direct current was administered via two $5 \times 5 \mathrm{~cm}$ pads (SnapPad), pre-saturated with a $0.9 \%$ saline solution $(9 \mathrm{~g}$ of salt per liter) and positioned using the OLE system corresponding to (10-20 international system) F3 Anode and F4 Cathode. The OLE montages maximizes anodal-directed current to IDPFC (Seibt et al., 2015) while stimulating across PFC and have been suggested optimal in prior trials of ABMT (Shahbabaie et al., 2018) and in trials of other anxiety-linked psychiatric disorders (Brunoni et al., 2017). The OLE approach, being automated by the head-gear (Seibt et al., 2015), also translates to home-use consistent with the broader deployable goals of our research program (Kasschau et al., 2016; Dobbs et al., 2018). The intensity of tDCS was $2 \mathrm{~mA}$ with a 30-s ramp up/down time. Stimulation began $\sim 2$ min before the initiation of ABMT to allow for acclimation to stimulation before training began. If participants reported adverse effects/discomfort that did not abate during the ramp-up period, the acclimation period was extended until discomfort alleviated, after which participants began ABMT while tDCS was simultaneously administered at $2 \mathrm{~mA}$ or the maximum intensity that participants indicated was tolerable. In the sham condition identical protocol was followed except stimulation current was ramped up (30-s) and down (30-s) at the beginning and end of the session. Because the researchers (trained personnel) were required to operate the tDCS device in order to deliver stimulation to participants properly, the design was single-blind, as only participants were blinded to whether they received the active or sham $\mathrm{tDCS}$.

\section{Mobile, Gamified Attention Bias Modification Training (ABMT)}

During the neuromodulation procedure, all participants received the active version of the ABMT app, commercially available under the name Personal Zen. Participants sat comfortably at a table and were given an iPod Touch or used their iOS device (e.g., iPhone) to practice the app to ensure understanding (see 


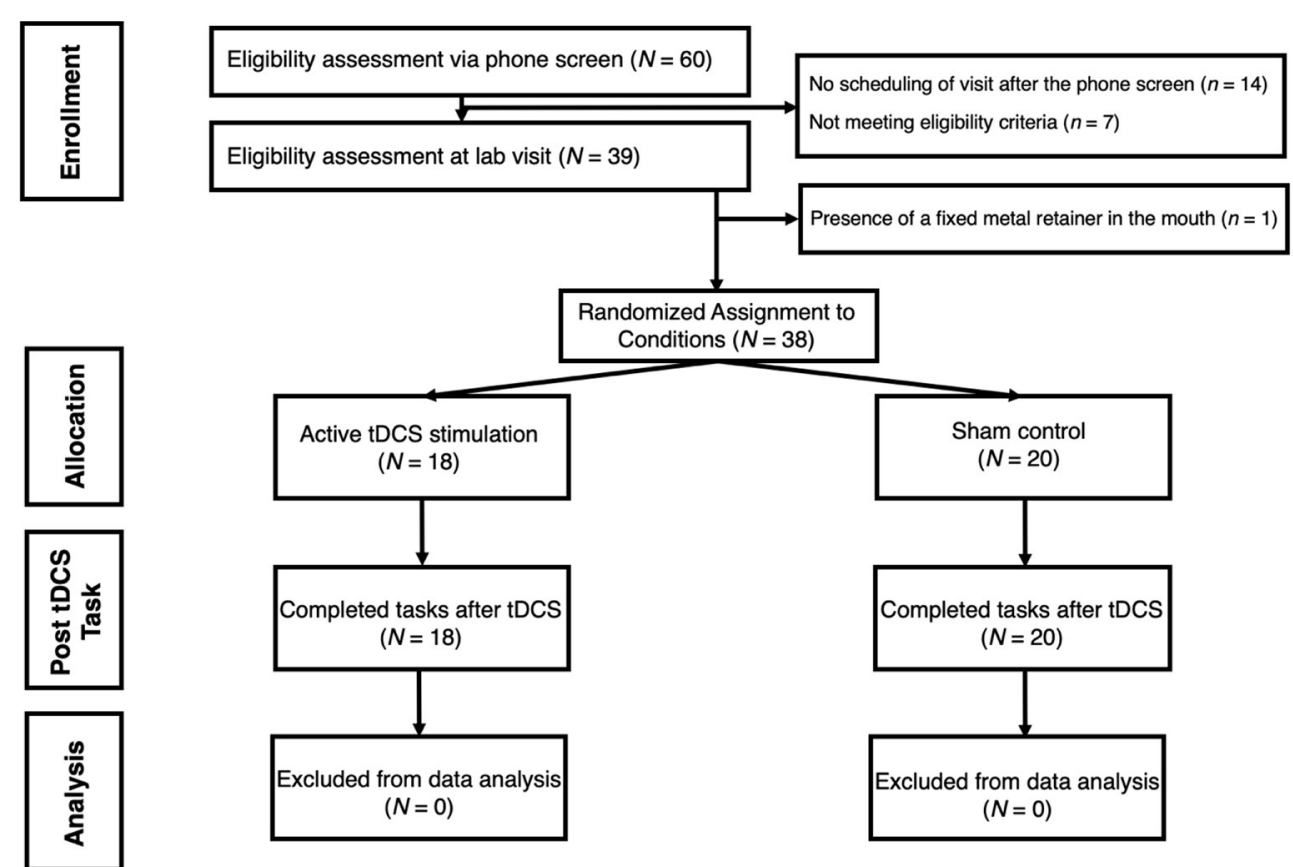

FIGURE 1 | Flowchart demonstrating participant selection process for the study. tDCS is for transcranial Direct Current Stimulation.

Dennis-Tiwary et al., 2017). The following instructions were provided: "In this attention training app, two animated characters will appear on the screen. Shortly after, they will burrow into a hole. One of them will cause a path of grass to rustle behind it. With your finger, trace the path of the rustling grass, beginning from the burrow. Trace the grass as smoothly, quickly, and accurately as possible. At no point should you feel rushed, you should be comfortable." Then, they were allowed to complete one practice round under the guidance of the experimenter who answered any questions about the app. For every trial, two cartoon characters (sprites), one showing an angry expression and one showing a neutral/mildly pleasant expression, appeared simultaneously on the screen for $500 \mathrm{~ms}$. Next, both sprites simultaneously "burrowed" into the grass field (see DennisTiwary et al., 2017 for images of the app; Dennis-Tiwary et al., 2016). Then, a trail of grass appeared in the location of the non-threat character for every trial. The grass remained until participants responded by correctly tracing the grass path starting from the point at which the sprite burrowed out of sight. Participants were instructed to play the app for two $12.5 \mathrm{~min}$ sessions ( $\sim 25$ min of app play total), separated by a 5-min break. Each session consisted of $\sim 40-45$ app rounds (varied based on user speed) with 12 trials per round. Number of training trials were consistent with previously documented effective "dosages" of the app (Dennis and O'Toole, 2014; Dennis-Tiwary et al., 2016, 2017).

\section{The Dot Probe}

The dot probe task (Mathews and Mackintosh, 1998; Bar-Haim et al., 2007) was administered immediately prior to and following administration of $\mathrm{tDCS} / \mathrm{ABMT}$ procedure to measure AB. The dot probe followed parameters of the Tel-Aviv University/National Institute of Mental Health protocol. Stimuli for the dot probe task are pictures of 20 different individuals (10 males, 10 females) from the NimStim stimulus set (Tottenham et al., 2009) with one female taken from the Matsumoto and Ekman (Matsumoto and Ekman, 1989) set. Stimuli were programmed using E-Prime version 2.0 (Schneider et al., 2002).

During each trial, two pictures were presented, either angryneutral face pairs or neutral-neutral face pairs (depicting the same individual). The pictures were shown above and below a fixation cross, with $14 \mathrm{~mm}$ between them. The task included 120 trials [80 threat (angry faces) and neutral faces (TN) and 40 non-threat both neutral faces $(\mathrm{NN})]$. Each trial comprised: (a) $500 \mathrm{~ms}$ fixation, (b) $500 \mathrm{~ms}$ face-pair cue, which then disappears, (c) probe (target) in the former location of one of the faces until a response is made via the left or right mouse button to indicate the direction in which the arrow is pointing, and (d) $500 \mathrm{~ms}$ intertrial interval. Participants were asked to respond as quickly and as accurately as possible whether the arrow was pointing to the left or the right. Probes were equally likely to appear on the top or bottom, in the location of the angry or neutral face cues and pointing to the left or the right.

\section{Quantifying Attention Bias (AB)}

$\mathrm{AB}$ was measured via the dot probe task. Dot probe trials with incorrect responses were excluded from further processing and analyses. Responses faster than $-2.5 S D$ from an individual's mean and slower than $+2.5 S D$ from an individual's mean were removed. The average response time was $535(S D=101)$ 
TABLE 1 | Descriptive statistics on self-reported questionnaires, attention bias, disengagement, and RSA.

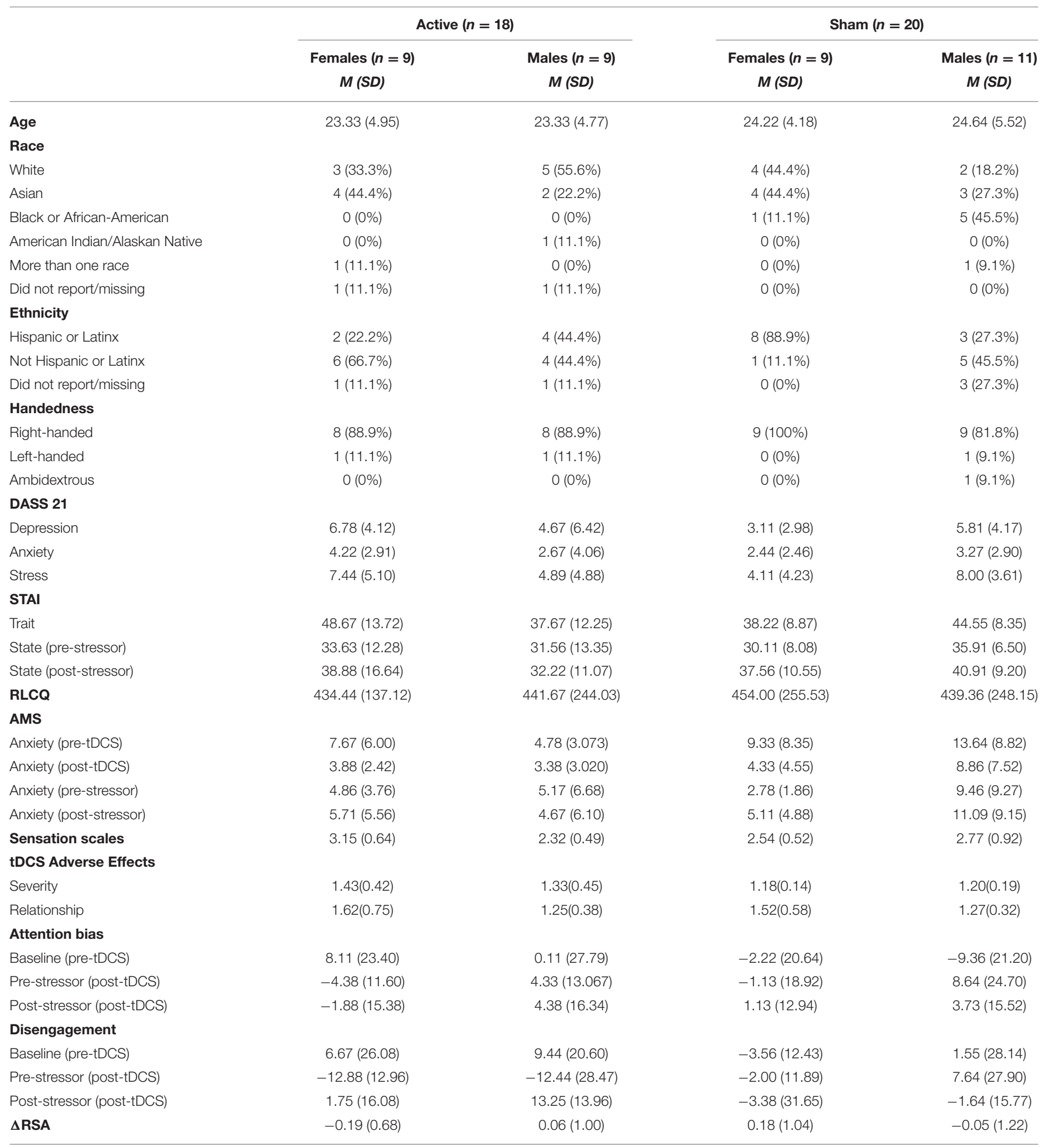

DASS-21, Depression Anxiety and Stress Scales; STAI, State-Trait Anxiety Inventory; RLCQ, Recent Life Changes Questionnaire; AMS, Analog Mood Scale; RSA, Respiratory sinus arrhythmia.

milliseconds and the overall accuracy rate prior to training was $0.99(S D=0.01)$. AB was calculated in two ways. First, to quantify overall attention capture by threat, a threat bias score was computed as the average RTs for neutral probes in TN trials minus RTs for angry probes in TN trials. Second, to quantify the more specific effortful top-down inhibition of attention, a 
difficulty disengaging score was computed as the average RTs for neutral probes in TN trial minus RTs for neutral probes in the $\mathrm{NN}$ trials.

\section{Anagrams Task}

Participants completed an anagrams task (Bishop, 2009) consisting of 40 medium to difficult mixed letter words (i.e., anagrams; e.g., RISECET $=$ RECITES). Fourteen of the anagrams were not solvable as real words (i.e., IUTRUCE). Participants received the following instructions verbatim: "For this task you will be asked to solve 40 anagrams. You will have $3 \mathrm{~min}$ to complete this task. When you have solved an anagram press the spacebar to proceed. Please do this task as quickly and as accurately as possible." Participants were also told that the solutions must be English words, and none of the solutions were proper nouns. Anagrams were presented on the computer and participants were asked to write down their solutions on a sheet of paper. After 3 min elapsed, participants received the following instructions: "Your time is up. Please hand your score sheet to the research assistant."

\section{Electrocardiogram (ECG) ECG Application}

ECG was recorded via a Biopac MP150 wireless system (Biopac Systems, CA, USA). Three sticker-based electrodes were applied, one to each clavicle and one on the left rib and were connected via three leads to a transmitter attached to a Velcro strap which participants wore around their waists. ECG data was wirelessly transmitted to a computer to allow for ambulatory recording with Acqknowledge v4.4 software. ECG was recorded continuously during the entire study session with the exception of questionnaire completion. Manual event-markers indicated the beginning and ending of each task.

\section{ECG Processing and RSA Quantification}

The ECG data was segmented during recording based on the onset and offset of the two baseline tasks and the anagrams tasks. Mindware 3.14 software was later used to process data, reject artifacts, and compute scores. Inter-beat intervals (IBI) were defined as the temporal distance between R-spikes, which represent the contraction of the ventricles of the heart. ECG recordings were segmented into 30 -s sections, which were each manually inspected for missing or incorrectly labeled R-spikes. Segments with $>10 \%$ artifacts were not included in computed scores, consistent with criteria used in previous studies (e.g., Blandon et al., 2008). Spectral analyses used a Hamming window, and heart rate variability (HRV) in the high-frequency band of spontaneous respiration in adults was targeted to quantify RSA $(0.120-0.420 \mathrm{~Hz})$ consistent with prior studies (e.g., Denver et al., 2007). RSA was calculated via Mindware software using the Porges (Porges, 1985) method which applies an algorithm resulting in natural log transformed variance in heart rate period while accounting for respiration in units of $\ln (\mathrm{ms})^{2}$. Finally, we examined sample-level change in autonomic arousal in response to the stressor (anagrams task) vs. baseline as a manipulation check to supplement the test of increase in subjective anxiety
(STAI-S). We generated low frequency HRV (LF-HRV, 0.040$0.120 \mathrm{~Hz}$ ) and quantified arousal via the ratio between low and high frequency (LF/HF). Prior research has documented an increase in LF/HF ratio during acute laboratory stressors (Castaldo et al., 2015).

RSA suppression ( $\triangle \mathrm{RSA}$ ) was quantified using residual scores, which have been used in prior studies comparing biological responses to emotional stimuli or events like a stressor (e.g., Myruski et al., 2017). Residual scores offer an advantage in comparison to subtraction scores such that residuals are more resistant to bias due to baseline inter-correlation (Weinberg et al., 2015). To quantify stress resilience for each participant, $\triangle$ RSA scores were computed by generating residuals with baseline RSA as the predictor and RSA in the anagrams task as the outcome. More negative $\triangle$ RSA scores indicated greater stress resilience, or greater ability to flexibly engage regulatory processes in the face of a challenge.

\section{Self-Report Measures tDCS Sensation Scales}

Participants completed the Sensation Scale (developed by the researchers) to rate their level of discomfort due to tDCS [ $1=$ no sensation; $2=$ slight sensation; $3=$ tingly; $4=$ slightly uncomfortable; $5=$ very uncomfortable]. For all participants, this scale was completed a minimum of four times as follows: First directly after application of the tDCS apparatus, again once stimulation reached $2 \mathrm{~mA}$ prior to the onset of the 30 -min stimulation period, again during a break approximately half-way through the 30-min stimulation period, and finally, after tDCS removal. For cases in which participants' initial sensation rating was "very uncomfortable" (5), additional Sensation Scales were administered as stimulation levels were adjusted or participants habituated to the stimulation sensation. If participants opted to reduce the level of stimulation, which was only done prior to the onset of the 30-min stimulation period, current was set to $1.5 \mathrm{~mA}$ and another Sensation Scale was administered after $60 \mathrm{~s}$. If sensation rating fell below "very uncomfortable" (5) at that point, stimulation was again increased to $2 \mathrm{~mA}$ to aim for uniformity in voltage across participants. In these cases, an additional Sensation Scale was administered after increasing stimulation back to $2 \mathrm{~mA}$. If participant rating remained at "very uncomfortable" (5), the study session was discontinued to avoid undue participant distress.

\section{The Depression, Anxiety, and Stress Scale (DASS-21)}

The DASS-21 (Henry and Crawford, 2005) is a 21-item questionnaire that measures the severity of symptoms across three domains: depression, anxiety, and stress. Each subscale contains 7 items, scored on a $0-3$ scale, and with scores ranging from 0 to 21 for each subscale. The anxiety subscale was used for the present study to evaluate the impact of individual differences anxiety symptoms on effects of tDCS combined with ABMT. A score of 4-5 indicates mild anxiety. Participants' anxiety scores ranged from 0 to 17 , with most (82\%) reporting normal levels of anxiety. The DASS-21 was used to measure anxiety, depression, and stress for study recruitment. 


\section{State Trait Anxiety Inventory (STAI)}

The STAI (Spielberger, 1983) is a 40-item questionnaire that assesses state (20 questions) and trait (20 questions) anxiety symptom severity. Respondents are asked to indicate the degree to which each statement reflects how they feel right now and in general using a Likert-type scale ranging from 1 (not at all) to 4 (very much so). The STAI yields total scores on two scales reflecting state and trait anxiety. The state anxiety score was used in the current study to evaluate changes in mood related to the stressor, and the trait anxiety score to assess individual differences in anxiety.

\section{Recent Life Changes Questionnaire (RLCQ)}

The RLCQ (Miller and Rahe, 1997) consists of 91 items listing different life events experienced in the past 12 months that can cause stress and assigns a numerical value (ranging from 18 to 123) to the level or magnitude of stress the event typically causes (e.g., a vacation receives a score of 24 , whereas the death of a spouse is scored 119). Scores for every item endorsed were summed and used in analyses below to examine the impact of life stress on effects of $\mathrm{tDCS}$ combined with ABMT.

\section{Analog Mood Scale (AMS)}

The AMS (MacLeod et al., 2002) is a brief measure of positive and negative mood consisting of three questions (i.e., "How anxious are you?" "How sad are you?" and "How happy are you?"). Participants were asked to indicate their present mood by identifying a location on a horizontal line divided into 30 equally sized sections labeled 1 (not at all) to 30 (very much). The AMS anxiety question was used in the current study to quantify changes in mood induced by tDCS administration and the anagrams task. Ratings were collected at baseline, following tDCS/ABMT administration, immediately prior to the stressor, and immediately following the stressor.

\section{tDCS Adverse Effects Questionnaire}

At the conclusion of the study session, participants completed the tDCS Adverse Effects Questionnaire (Brunoni et al., 2011) regarding their current physical, cognitive, and emotional state. Participants reported the severity of 10 adverse effects such as headache, burning sensation, trouble concentrating, and acute mood change on a scale from 1 (absent) to 4 (severe). If an adverse effect was present [rating of 2 (mild) and above], participants reported the degree of relatedness the effect had to tDCS stimulation on a scale from (1) none to (5) definite.

\section{RESULTS}

\section{Descriptive Statistics}

Table 1 presents descriptive statistics for study variables, separately for each tDCS group (active, sham). No baseline group differences reached significance, and tDCS groups were similar in age and gender. Further, there were no significant differences between the active and sham groups regarding in adverse events ( $p$ 's $>0.05)$.

Table 2 shows correlations among study variables (DASSAnxiety, dot probe attention bias, dot probe disengagement, state anxiety, RSA at baseline, and baseline AMS). Measures of anxiety were significantly positively inter-correlated. Threat bias was not significantly correlated with any measures of anxiety, but RSA at baseline was significantly negatively correlated with post-stressor state anxiety.

\section{Dot Probe Reliability}

Split-half reliability was examined by generating mean RTs by condition (neutral probes in TN trials, angry probes in TN trials, neutral probes in NN trials) and $\mathrm{AB}$ scores (threat bias, difficulty disengaging), separately for even and odd trials for each dot probe task (before and after tDCS/ABMT). Pearson correlations were conducted between even and odd versions of each mean RT and AB score metric. Spearman-Brown correction $\left(r_{s b}\right)$ was applied to correlation coefficients to account for fewer (half) trials used to compute RT means, with corrected $r$ values above 0.70 indicating acceptable reliability. For the both dot probe tasks, strong and significant correlations emerged for all even and odd mean RT associations [first dot probe task: neutral probes in TN trials $\left(r=0.96, p<0.001, r_{s b}=0.98\right)$, angry probes in TN trials $\left(r=0.97, p<0.001, r_{s b}=0.98\right)$, neutral probes in NN trials $\left(r=0.96, p<0.001, r_{s b}=0.98\right)$; second dot probe task: neutral probes in TN trials $\left(r=0.97, p<0.001, r_{s b}=0.98\right)$, angry probes in TN trials $\left(r=0.86, p<0.001, r_{s b}=0.92\right)$, neutral probes in $\mathrm{NN}$ trials $\left.\left(r=0.98, p<0.001, r_{s b}=0.99\right)\right]$. However, AB scores derived separately from even and odd trials were not significantly correlated for the first dot probe [threat bias $(r=0.06, p=0.77$, $\left.r_{s b}=0.11\right)$, difficulty disengaging $\left.\left(r=0.24, p=0.26, r_{s b}=0.39\right)\right]$. For the second dot probe, difficulty disengaging $\mathrm{AB}$ score did show significant correlation across split halves $(r=0.47, p=0.03$, $\left.r_{s b}=0.64\right)$, but threat bias did not $\left(r=-0.30, p=0.18, r_{s b}=\right.$ $-0.86)$. This poor reliability of $\mathrm{AB}$ measures is consistent with the previous literature (Schmukle, 2005; Kappenman et al., 2014; Rodebaugh et al., 2016).

\section{Manipulation Check: Stressor Effects}

To confirm that the anagrams task induced subjective anxiety, we conducted a simple within-subjects paired $t$-test between state anxiety (STAI-state) assessed immediately following the stressor $(M=37.54, S D=11.85)$ compared to state anxiety immediately prior to the stressor $[(M=32.95, S D=10.03), t(36)$ $=-3.35, p=0.002 ; d=0.55]$ and at baseline $[(M=33.33$, $S D=10.39), t(36)=-2.36, p=0.024 ; d=0.39$ ]. Both $t$-tests reached significance while controlling for multiple comparisons (Bonferroni's adjusted $p=0.025$ ), confirming the induction of anxiety. A paired $t$-test was also conducted to examine changes in HRV (LF/HF ratio), however no significant change in HRV was detected following the stressor $(M=10.89, S D=2.12)$ in comparison to baseline $(M=11.28, S D=3.57), t(34)=1.39$, $p=0.173 ; d=0.24$.

\section{Manipulation Check: ABMT Effects Independent of tDCS}

To test whether ABMT, independent of tDCS, reduced two key targets of $\mathrm{ABMT}-\mathrm{AB}$ (threat bias and difficulty disengaging) and subjective anxiety (AMS-anxiety) - we conducted two 2(Time: pre, post $) \times 2$ (Sex: male, female) repeated measures ANOVAs 
TABLE 2 | Correlations between scores of self-reported questionnaires, Attention Bias (AB), Disengagement (DIS), and Respiratory Sinus Arrhythmia (RSA) separated by time points.

\begin{tabular}{|c|c|c|c|c|c|c|c|c|c|c|c|c|c|c|}
\hline & 1 & 2 & 3 & 4 & 5 & 6 & 7 & 8 & 9 & 10 & 11 & 12 & 13 & 14 \\
\hline 1. DASS-A & - & & & & & & & & & & & & & \\
\hline \multicolumn{15}{|l|}{ Baseline } \\
\hline 3. STAI-S & $0.34^{\star}$ & $0.83^{\star \star}$ & - & & & & & & & & & & & \\
\hline 4. $A B$ & -0.11 & -0.20 & 0.16 & - & & & & & & & & & & \\
\hline \multicolumn{15}{|c|}{ Pre-stressor } \\
\hline 7. AMS-A & $0.45^{\star \star}$ & 0.18 & 0.14 & -0.21 & 0.09 & -0.30 & - & & & & & & & \\
\hline 8. STAI-S & $0.69^{* \star}$ & $0.43^{\star \star}$ & $0.53^{\star \star}$ & -0.08 & -0.04 & -0.09 & $0.60^{\star \star}$ & - & & & & & & \\
\hline 9. $A B$ & -0.05 & 0.08 & 0.05 & 0.07 & 0.27 & 0.045 & -0.01 & -0.01 & - & & & & & \\
\hline 10. DIS & 0.33 & 0.05 & 0.03 & -0.09 & 0.11 & -0.01 & 0.26 & $0.34^{*}$ & $0.46^{\star *}$ & - & & & & \\
\hline 14. DIS & 0.11 & -0.08 & -0.08 & -0.28 & 0.04 & -0.06 & -0.05 & -0.03 & -0.04 & 0.06 & -0.18 & -0.12 & $0.58^{\star \star}$ & \\
\hline
\end{tabular}

A, Anxiety; S, State; ${ }^{*} p<0.05 .{ }^{* *} p<0.01$.

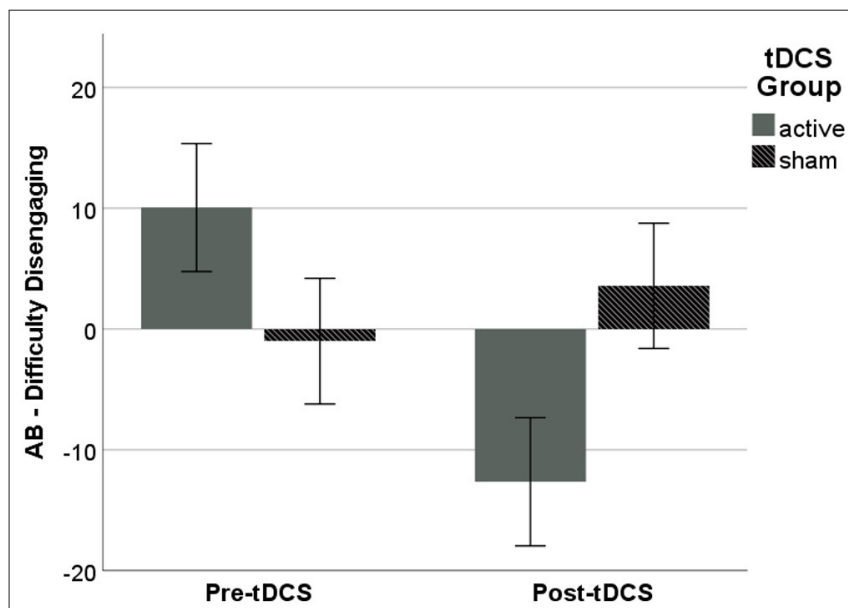

FIGURE 2 | AB following ABMT, as measured via difficulty disengaging from threat, was significantly lower in the active vs. sham Group. Error bars represent \pm 1 SE.

separately for each of the three DVs. Bonferroni's correction was applied to control for multiple comparisons for follow-up tests.

There was a significant effect of Time on AMS-anxiety, $F_{(1,34)}=9.95, p=0.003, \eta_{p}{ }^{2}=0.226$, showing reduced subjective anxiety over time in the sample as a whole (pre: $M=9.11$, $S D=7.56$; post: $M=5.40, S D=5.43$ ).

No significant effects emerged for $\mathrm{AB}$ metrics $\left(p^{\prime} s>0.10\right)$.

\section{Effects of tDCS Combined With ABMT}

To test the main study hypothesis that participants receiving tDCS across the PFC, compared to sham tDCS, will show augmented benefits of $A B M T$, measured via reduced $A B$, reduced anxious mood (AMS- anxiety), and enhanced stress resilience (greater RSA suppression), we conducted two 2 (tDCS Group: active vs. sham $) \times 2$ (Sex: male, female) ANCOVAs with pretDCS measures of each DV as the covariate, separately for each $\mathrm{AB}$ score (threat bias and difficulty disengaging) and AMSanxiety. Because RSA suppression is calculated as a differences score using residuals scores involving baseline RSA, baseline RSA was not used as a covariate, and an ANOVA with tDCS Group and Sex as the between-subjects factors was conducted instead for RSA as the dependent variable. Bonferroni's correction was applied to control for multiple comparisons for follow-up tests.

The main effect of $\mathrm{tDCS}$ Group, $F_{(1,31)}=5.18, p=0.030$, $\eta_{p}{ }^{2}=0.143$, showed that $\mathrm{AB}$, measured as difficulty disengaging, following ABMT was significantly lower in the active vs. sham Group, (active: $M=-12.65, S D=21.88$; sham: $M=3.58$, $S D=22.62 ; p=0.030, \eta_{p}^{2}=0.127$; Figure 2$)$. There were no significant effects on other metrics of $\mathrm{AB}$ ( $p$ 's $>0.10$ ), and analyses with subjective anxiety and RSA suppression as the dependent variables did not reach significance ( $p$ 's $>0.10$ ).

\section{Exploratory Analyses of Moderators of Efficacy}

To further examine individual differences in the impact of tDCS combined with ABMT on target outcomes, we conducted six hierarchical linear regressions via the SPSS PROCESS macro to test for the moderating effect of trait anxiety (STAI trait) and life stress (recent life changes over the past year) on the DVs (AMSanxiety, AB, and RSA suppression). Step 1 was tDCS Group (active, sham); Step 2 was the moderator (recent life stressor or trait anxiety scores); and Step 3 was the interaction between the two. For AMS-anxiety and AB, pre-stressor baseline measures 
TABLE 3 | Regression model - effects of group and life stress on RSA suppression.

\begin{tabular}{|c|c|c|c|c|c|c|}
\hline & $b$ & $S E B$ & $B$ & $R^{2}$ & $\Delta R^{2}$ & $p$ \\
\hline Model & & & & 0.44 & & 0.086 \\
\hline Step 1 & & & & 0.004 & 0.004 & \\
\hline Group & 0.13 & 0.55 & 0.064 & & & 0.71 \\
\hline Step 2 & & & & 0.006 & 0.002 & \\
\hline Group & 0.13 & 0.34 & 0.065 & & & 0.72 \\
\hline Group & 1.86 & 0.73 & 0.95 & & & 0.016 \\
\hline RLS & 0.01 & 0.003 & 1.43 & & & 0.020 \\
\hline Group $\times$ RLS & -0.004 & 0.001 & -1.78 & & & 0.013 \\
\hline
\end{tabular}

RLS, Recent Life Stressor:

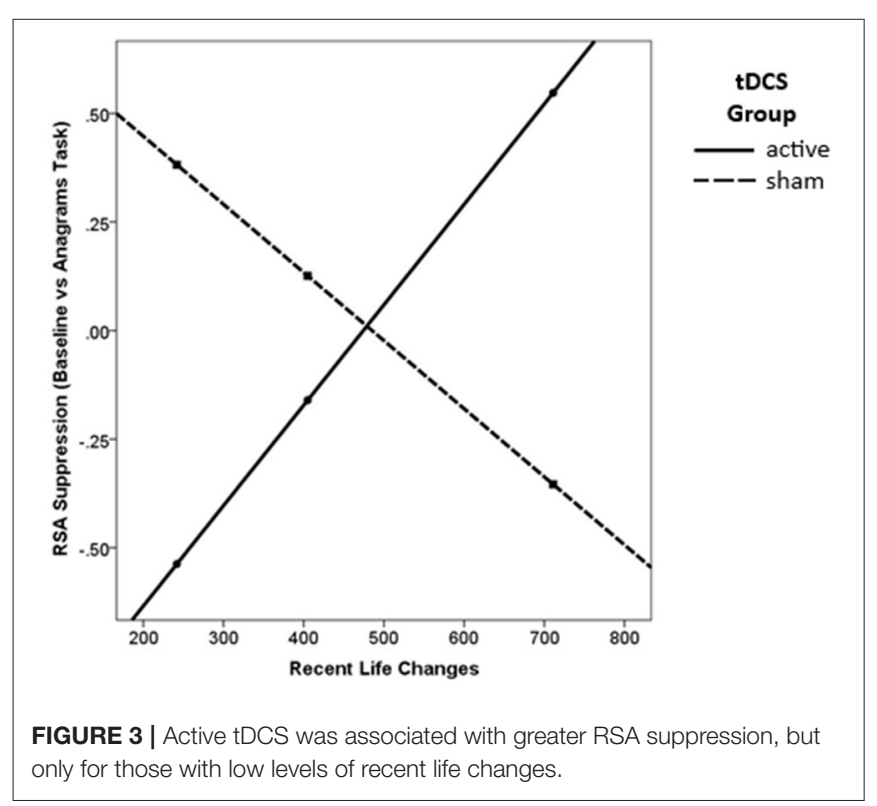

were included in the model as covariates. To account for multiple comparisons, the Benjamini-Hochberg procedure (Benjamini and Hochberg, 1995) was applied. All p-values reported below are raw and were significant using a false discovery rate of 0.10 .

There was a significant main effect of tDCS Group on RSA suppression, such that those in the active group showed significantly greater RSA suppression overall $[\beta=0.95, p=0.016$; Table 3]. Lower levels of recent stressful life events also significantly predicted greater RSA suppression $[\beta=1.43, p=0.020]$ for the sample as a whole. Finally, there was a significant interaction $\left[R^{2}\right.$ change $=0.18, p=0.013]$ such that active tDCS was associated with increased RSA suppression for low levels of recent stressful life events $[\beta=0.92, t(31)=2.11, p=0.043$, Figure 3].

No other regression analyses reached significance (model $p$ 's $>0.10$ ).

\section{Exploratory Analyses of AB Mediation of Anxiety}

We also conducted mediation analyses via the SPSS PROCESS macro to test whether $\mathrm{AB}$ (threat bias and difficulty disengaging) mediated the association between tDCS Group (active vs. sham) and outcomes (AMS-anxiety and RSA suppression). No models reached significance (model $p$ 's $>0.10$ ).

\section{DISCUSSION}

The current study tested whether tDCS across the PFC augmented the efficacy of mobile, gamified ABMT for anxietyrelated $\mathrm{AB}$. It extends previous studies by using an alternative delivery system for AMBT, a mobile, gamified app, and by identifying underlying mechanisms that drive individual difference in response to ABMT. In the sample as a whole, ABMT reduced subjective anxiety after, compared to before, app play. While this may indicate a beneficial effect of the app, these findings should be interpreted with caution because there was no non-ABMT control group to confirm this interpretation. Consistent with our predictions, adding tDCS (compared to sham) reduced $\mathrm{AB}$ measured as difficulty disengaging attention from threat, but neither of the other two main outcomes (anxiety, stress resilience) were significantly different between groups. Exploratory analyses showed that combined tDCS and ABMT boosted stress resilience measured via RSA suppression, particularly among those reporting lower life stress. Results provide important experimental evidence for potential mechanisms of ABMT, and advance our understanding and identification of treatment moderators, both of which support the aim of developing more personalized treatment approaches for anxiety.

Following early enthusiasm for ABMT, due to robust effect sizes emerging from well-controlled randomized clinical trials (Hakamata et al., 2010), subsequent RCTs showed mixed and null findings (Kruijt et al., 2019), leading to significant debate about the clinical utility of $\mathrm{AB}$ (Emmelkamp, 2012) and the potential heterogeneity of both $A B$ and of ABMT response (Dennis-Tiwary et al., 2019). Our study documented that targeted stimulation of the PFC in combination with ABMT specifically reduced 
difficulty disengaging from threat, an aspect of $\mathrm{AB}$ closely linked to inhibitory cognitive control processes, but not threat bias, which is more closely linked to attention capture by threat (Cisler and Koster, 2010). This differential finding strengthens the evidence base that changes in PFC-mediated cognitive control processes may underlie the positive effects of ABMT, particularly on $\mathrm{AB}$.

Current findings are also in line with previous data suggesting that PFC activation may modulate difficulty disengaging attention from threat among high-anxious individuals. For instance, highly trait-anxious individuals reporting poor attention control (as a proxy of the reduced PFC activity) exhibit more delayed disengagement from threat (Derryberry and Reed, 2002). Consistently, at the neural level, cortical structures centered around the pre-frontal cortex and its functionally related structures (i.e., anterior cingulate cortex and orbitofrontal cortex) may mediate delayed disengagement from threat through individual differences in the ability to down-regulate the influence of limbic structures and maintain attention on task-relevant stimuli (Bishop, 2009; Blair et al., 2012). This hypothesis makes sense in the context of previous work demonstrating that the activation of the PFC is functionally related to a down-regulation of amygdala activity during the presentation of threatening stimuli (Bishop et al., 2004). Future studies should examine the impact of tDCS across the PFC during ABMT using neuroimaging techniques such as fMRI to explore whether such activation is associated with modulation of PFC-amygdala connectivity during the dot-probe task or of specific regions of the cortex, such as the lateral PFC (Browning et al., 2010).

As predicted, we also found that tDCS combined with ABMT led to greater RSA suppression in response to a stressor. Larger magnitude RSA suppression is considered an adaptive physiological response reflecting the ability to flexibly shift between parasympathetic and sympathetic nervous system engagement in the service of coping with an emotional challenge. This finding is novel and suggests that downstream indices of affective regulation may be impacted directly by tDCS. In particular, bifrontal tDCS may target cortical pathways responsible for descending control of the autonomic nervous system, which include the insular cortex and anterior cingulate cortex as identified in human imaging studies (see Cechetto, 2014 for a review).

Importantly, these effects were particularly robust among those reporting low levels of current life stress. In contrast, active tDCS did not predict greater RSA suppression among those with relatively high recent life stress, potentially due to the deleterious effect of life stressors both severe (e.g., Valerio, 2004) and moderate (Jarczok et al., 2013) on ANS activity which may have overshadowed any bolstering effects of tDCS. This finding points to the need for more research exploring the impact of treatment moderators, both general and specific to either ABMT or tDCS. Such an evidence base is a necessary first step toward truly personalized treatment approaches and clinical specificity in identifying those who are most likely to benefit from both treatment approaches (Heeren et al., 2015; Price et al., 2018; Dennis-Tiwary et al., 2019).
This study was unique in its use of mobile, gamified ABMT combined with tDCS, which cannot be directly compared with prior studies combining tDCS and ABMT (Clarke et al., 2014; Heeren et al., 2015). However, the evidence base for this particular app is promising (Dennis and O'Toole, 2014; Dennis-Tiwary et al., 2016, 2017; Sprunger, 2018), and with the growth of evidence-based digital therapeutics that reduce barriers to treatment access (Kazdin and Blase, 2011; Kazdin and Rabbitt, 2013), it remains a key goal to empirically test the generalizability of digital mental health interventions, including mobile applications. Empirical support for gamified ABMT and tDCS is particularly relevant to inform future investigations of home-based interventions. Recent research has supported the utility of adapted tDCS protocols for self-administered use at home (Brietzke et al., 2020; Stein et al., 2020), further lowering barriers to treatment accessibility.

In addition to the relatively small sample size, and challenges in the reliable assessment of AB (e.g., Waechter and Stolz, 2015), several limitations should be noted. First, while our stressor manipulation check showed that subjective anxiety significantly increased due to the anagrams task, sample-level HRV did not significantly increase. This pattern could be due to differences in stress resilience (RSA suppression) induced by tDCS combined with ABMT, which may have reduced our ability to detect sample-level change as measured by the LF/HF ratio. Next, while tDCS was compared to sham stimulation, ABMT was not compared to a placebo control. While this limited our ability to examine interactions between ABMT and tDCS, we were able to demonstrate that across both tDCS conditions, subjective anxiety was reduced over time (after vs. before using the app), consistent with previous studies (Dennis and O'Toole, 2014; Dennis-Tiwary et al., 2016, 2017). This suggests that regardless of neurocognitive changes induced by tDCS, ABMT may reduce subjective negative mood specifically, but future research with a placebo control is needed. Moreover, we did not investigate the effects of tDCS without ABMT, and thus cannot rule out the possibility that $\mathrm{tDCS}$ alone, without $\mathrm{ABMT}$, reduces $\mathrm{AB}$ and promotes RSA suppression. Relevant to this question, however, a prior study (Coussement et al., 2019) tested whether tDCS alone induced plasticity in $\mathrm{AB}$, reasoning that strengthening cognitive control via tDCS might directly reduce $A B$, which is thought to be characterized by inadequate cognitive control. This study reported null findings, suggesting that tDCS alone is not sufficient to modify AB. On the other hand, participants in this study, much like the present study and a prior study combining tDCS and ABMT (Clarke et al., 2014), did not exhibit elevated AB at baseline, which may indicate that the presence of significant $A B$ is a necessary condition for $A B$ plasticity especially in the absence of interventions intended to train $A B$, such as $A B M T$ (e.g., Mogoaşe et al., 2014; Heeren et al., 2015). Indeed, findings of Clarke and colleagues (Clarke et al., 2014) showed an effect of tDCS on training to avoid threat and to attend to threat (the latter at the level of a trend), indicating that tDCS may more broadly influence the modification of both $\mathrm{AB}$ toward and away from threat. Thus, results should be interpreted with caution and future research could benefit from including both control conditions simultaneously for ABMT and $\mathrm{tDCS}$, to directly cross 
both the presence/absence of ABMT with tDCS, and to examine the effects of each alone.

While $A B$ assessment has been widely used to assess threat bias with substantial prior literature supporting the validity of the measure, the psychometric properties of these $\mathrm{AB}$ measures has started to be re-evaluated for their low internal consistency (Kappenman et al., 2014) and test-retest reliability (Schmukle, 2005; Rodebaugh et al., 2016; Molloy and Anderson, 2020). However, in the current study, one AB score, difficulty disengaging measured post-ABMT, did show significant split-half reliability, and this was the outcome which yielded significant main effect of tDCS Group (active vs. sham). This highlights that reliable behavioral measures may be a critical prerequisite to detecting effects of combined tDCS and ABMT on $\mathrm{AB}$.

A further limitation is that the current design was singlenot double-blind, and we did not include a procedure to ask participants to guess whether they received active or sham stimulation. We did, however, confirm that adverse effects did not significantly differ between groups. We also did not compare distinct tDCS montages, as has been done in prior research which compared anodal stimulation across the left vs. right dlPFC (Heeren et al., 2015), or examine alternative position of the reference electrode (cathode), which may influence the current flow pattern through the brain (Bikson et al., 2010). While high-definition tDCS [HD-tDCS; (Datta et al., 2009)] allows targeting of specific cortical regions such as IDLPFC (Nikolin et al., 2015; Shen et al., 2016; Hill et al., 2018; Martínez-Pérez et al., 2019), we adopted an approach activating PFC which is broadly implicated in ABMT, and also to support future homeuse of tDCS (Shaw et al., 2017) in combination with mobile ABMT. Further, other neurostimulation techniques using lowintensity transcranial electrical stimulation (e.g., Guleyupoglu et al., 2013) such as transcranial pulsed current stimulation (tPCS), transcranial alternating current stimulation (tACS), and transcranial random noise stimulation (tRNS) are presumed to engage distinct biophysical targets. We selected tDCS for the present study since it has been most rigorously examined in comparison to tRNS and tACS (Elmasry et al., 2015), and has been the focus of the few ABMT studies using neurostimulation (Clarke et al., 2014; Heeren et al., 2015). However, future investigations could examine differences across these techniques in terms of enhancement of ABMT efficacy.

Because tDCS is thought to boost learning (O'Shea et al., 2014; Buch et al., 2017; Kronberg et al., 2020), it is important to distinguish between effects of tDCS on acute states (such as stress) and effects related to treatment such as ABMT. That is, it may be that $\mathrm{tDCS}$ boosts response only if an individual is already primed to benefit from a particular treatment (Bikson and Rahman, 2013), making tDCS an enhancer of responsiveness. A complementary question about the enhancement perspective on tDCS is whether moderators - such as anxiety and life stress explored in the current study - serve as general predictors of who responds to ABMT, and thus will also predict who responds best to tDCS combined with ABMT. On the other hand, it is unknown whether some moderators specifically that predict relative benefit of active tDCS vs. sham, rather than overall responsiveness. Identification of the latter would represent a key inclusion criterion in a pivotal trial of tDCS vs. sham.

Another limitation was that participants evidenced on average low to moderate levels of anxiety and received only a single session of ABMT. Both of these factors may have reduced our ability to detect additional effects of ABMT, which has shown mixed efficacy across studies with different populations and methods (e.g., Cristea et al., 2015) and may be most effective when administered over multiple, weekly sessions for clinically relevant elevations in $\mathrm{AB}$ and anxiety (e.g., Hakamata et al., 2010). Lower levels of anxiety severity, along with the relatively small sample size, might also have limited our ability to detect whether changes in $\mathrm{AB}$ mediated the effects of $\mathrm{tDCS}$ on target outcomes. Moreover, while $\mathrm{AB}$ is evidenced in both clinical and non-clinical anxiety (Bar-Haim et al., 2007), much of the compelling evidence for ABMT efficacy includes patients diagnosed with social anxiety or generalized anxiety disorders (Hakamata et al., 2010; Mogoaşe et al., 2014). Thus, it remains unclear whether current study findings can generalize to clinical populations. Moreover, the relatively low-to-moderate levels of anxiety severity in the current sample may have also limited our ability to detect moderating effects of anxiety on the efficacy of ABMT and/or tDCS. At the same time, by documenting the potential for tDCS to augment effects of ABMT even among those with relatively few anxiety symptoms, current findings suggests that ABMT can be enhanced to be broadly effective across the full spectrum of anxiety. Future research should include a broader range of anxiety severity including the clinical range, and explore additional moderators of efficacy.

Taken together, findings document that tDCS across the PFC combined with mobile $\mathrm{ABMT}$ reduced $\mathrm{AB}$ measured as difficulty disengaging attention from threat and boosted stress resilience measured via RSA suppression, particularly among those reporting lower life stress. Results lay the groundwork for crucial assessments of dose response parameters and more targeted examination of treatment mechanisms, as well as the identification of treatment moderators and the development of more personalized treatment approaches.

\section{DATA AVAILABILITY STATEMENT}

The raw data supporting the conclusions of this article will be made available by the authors, without undue reservation.

\section{ETHICS STATEMENT}

The studies involving human participants were reviewed and approved by Hunter College, The City University of New York. The patients/participants provided their written informed consent to participate in this study.

\section{AUTHOR CONTRIBUTIONS}

TD-T and MB developed the study concept and design. SM and HC performed data collection. TD-T and SM performed data analyses and interpretation. TD-T, SM, and HC drafted the paper 
and $\mathrm{MB}$ provided critical revisions. All authors approved the final version of the paper for submission.

\section{FUNDING}

This research was made possible by the following funding sources granted to Tracy Dennis-Tiwary: National Institute of Mental Health (R56MH111700, RF1MH120846) and the National Center for Advancing Translational Sciences of the National Institutes of Health (TR000457). This research was also made possible by funding sources granted to Marom Bikson: National Institutes of Health (NIH-NIMH 1R01MH111896, NIH-NIMH 1R01MH109289; NIH-NINDS 1R01NS101362).

\section{REFERENCES}

Barak, A., Hen, L., Boniel-Nissim, M., and Shapira, N. a. (2008). A comprehensive review and a meta-analysis of the effectiveness of internetbased psychotherapeutic interventions. J. Technol. Human Serv. 26, 109-160. doi: 10.1080/15228830802094429

Bar-Haim, Y., Lamy, D., Pergamin, L., Bakermans-Kranenburg, M. J., and Van Ijzendoorn, M. H. (2007). Threat-related attentional bias in anxious and nonanxious individuals: a meta-analytic study. Psychol. Bull. 133:1. doi: 10.1037/0033-2909.133.1.1

Benjamini, Y., and Hochberg, Y. (1995). Controlling the false discovery rate: a practical and powerful approach to multiple testing. J. R Stat. Soc. B 57, 289-300. doi: 10.1111/j.2517-6161.1995.tb02031.x

Bikson, M., Datta, A., Rahman, A., and Scaturro, J. (2010). Electrode montages for tDCS and weak transcranial electrical stimulation: role of "return" electrode's position and size. Clin. Neurophysiol. 121:1976. doi: 10.1016/j.clinph.2010.05.020

Bikson, M., Grossman, P., Thomas, C., Zannou, A. L., Jiang, J., Adnan, T., et al. (2016). Safety of transcranial direct current stimulation: evidence based update 2016. Brain Stimul. 9, 641-661. doi: 10.1016/j.brs.2016.06.004

Bikson, M., and Rahman, A. (2013). Origins of specificity during tDCS: anatomical, activity-selective, and input-bias mechanisms. Front. Human Neurosci. 7:688. doi: 10.3389/fnhum.2013.00688

Bishop, S., Duncan, J., Brett, M., and Lawrence, A. D. (2004). Prefrontal cortical function and anxiety: controlling attention to threat-related stimuli. Nat. Neurosci. 7, 184-188. doi: 10.1038/nn1173

Bishop, S. J. (2009). Trait anxiety and impoverished prefrontal control of attention. Nat. Neurosci. 12:92. doi: 10.1038/nn.2242

Blair, K. S., Geraci, M., Smith, B. W., Hollon, N., DeVido, J., Otero, M., et al. (2012). Reduced dorsal anterior cingulate cortical activity during emotional regulation and top-down attentional control in generalized social phobia, generalized anxiety disorder, and comorbid generalized social phobia/generalized anxiety disorder. Biol. Psychiatry 72, 476-482. doi: 10.1016/j.biopsych.2012.04.013

Blandon, A. Y., Calkins, S. D., Keane, S. P., and O'Brien, M. (2008). Individual differences in trajectories of emotion regulation processes: the effects of maternal depressive symptomatology and children's physiological regulation. Dev. Psychol. 44:1110. doi: 10.1037/0012-1649.44.4.1110

Bradley, B. P., Mogg, K., and Millar, N. H. (2000). Covert and overt orienting of attention to emotional faces in anxiety. Cogn. Emot. 14, 789-808. doi: 10.1080/02699930050156636

Brietzke, A. P., Zortea, M., Carvalho, F., Sanches, P. R., Danton Jr, P., da Silva Torres, I. L., et al. (2020). Large treatment effect with extended home-based transcranial direct current stimulation over dorsolateral prefrontal cortex in fibromyalgia: a proof of concept sham-randomized clinical study. J. Pain 21, 212-224. doi: 10.1016/j.jpain.2019.06.013

Browning, M., Holmes, E. A., Murphy, S. E., Goodwin, G. M., and Harmer, C. J. (2010). Lateral prefrontal cortex mediates the cognitive modification of attentional bias. Biol. Psychiatry 67, 919-925. doi: 10.1016/j.biopsych.2009.10.031

Brunoni, A. R., Amadera, J., Berbel, B., Volz, M. S., Rizzerio, B. G., and Fregni, F. (2011). A systematic review on reporting and assessment of adverse effects associated with transcranial direct current stimulation. Int. J. Neuropsychopharmacol. 14, 1133-1145. doi: 10.1017/S1461145710001690

Brunoni, A. R., Moffa, A. H., Sampaio-Junior, B., Borrione, L., Moreno, M. L., Fernandes, R. A., et al. (2017). Trial of electrical direct-current therapy versus escitalopram for depression. N. E. J. Med. 376, 2523-2533. doi: 10.1056/NEJMoa1612999

Buch, E. R., Santarnecchi, E., Antal, A., Born, J., Celnik, P. A., Classen, J., et al. (2017). Effects of tDCS on motor learning and memory formation: a consensus and critical position paper. Clin. Neurophysiol. 128, 589-603. doi: 10.1016/j.clinph.2017.01.004

Bystritsky, A. (2006). Treatment-resistant anxiety disorders. Mol. Psychiatry 11, 805-814. doi: 10.1038/sj.mp.4001852

Castaldo, R., Melillo, P., Bracale, U., Caserta, M., Triassi, M., and Pecchia, L. (2015). Acute mental stress assessment via short term HRV analysis in healthy adults: a systematic review with meta-analysis. Biomed. Signal Process. Control 18, 370-377. doi: 10.1016/j.bspc.2015.02.012

Cechetto, D. F. (2014). Cortical control of the autonomic nervous system. Exp. Physiol. 99, 326-331. doi: 10.1113/expphysiol.2013.075192

Charvet, L. E., Kasschau, M., Datta, A., Knotkova, H., Stevens, M. C., Alonzo, A., et al. (2015). Remotely-supervised transcranial direct current stimulation (tDCS) for clinical trials: guidelines for technology and protocols. Front. Syst. Neurosci. 9:26. doi: 10.3389/fnsys.2015.00026

Cisler, J. M., and Koster, E. H. (2010). Mechanisms of attentional biases towards threat in anxiety disorders: an integrative review. Clin. Psychol. Rev. 30, 203-216. doi: 10.1016/j.cpr.2009.11.003

Clarke, P. J., Browning, M., Hammond, G., Notebaert, L., and MacLeod, C. (2014). The causal role of the dorsolateral prefrontal cortex in the modification of attentional bias: evidence from transcranial direct current stimulation. Biol. Psychiatry 76, 946-952. doi: 10.1016/j.biopsych.2014. 03.003

Coussement, C., Maurage, P., Billieux, J., and Heeren, A. (2019). Does change in attention control mediate the impact of tDCS on attentional bias for threat? Limited evidence from a double-blind sham-controlled experiment in an unselected sample. Psychol. Belgica 59:16. doi: 10.5334/pb.449

Cristea, I. A., Kok, R. N., and Cuijpers, P. (2015). Efficacy of cognitive bias modification interventions in anxiety and depression: meta-analysis. $\mathrm{Br} . \mathrm{J}$. Psychiatry 206, 7-16. doi: 10.1192/bjp.bp.114.146761

Datta, A., Bansal, V., Diaz, J., Patel, J., Reato, D., and Bikson, M. (2009). Gyriprecise head model of transcranial direct current stimulation: improved spatial focality using a ring electrode versus conventional rectangular pad. Brain Stimul. 2, 201-207. doi: 10.1016/j.brs.2009.03.005

den Uyl, T. E., Gladwin, T. E., Lindenmeyer, J., and Wiers, R. W. (2018). A clinical trial with combined transcranial direct current stimulation and attentional bias modification in alcohol-dependent patients. Alcoholism 42, 1961-1969. doi: 10.1111/acer.13841

Dennis, T. A., and O'Toole, L. J. (2014). Mental health on the go: effects of a gamified attention-bias modification mobile application in trait-anxious adults. Clin. Psychol. Sci. 2, 576-590. doi: 10.1177/2167702614522228

Dennis-Tiwary, T. A., Denefrio, S., and Gelber, S. (2017). Salutary effects of an attention bias modification mobile application on biobehavioral measures of stress and anxiety during pregnancy. Biol. Psychol. 127, 148-156. doi: 10.1016/j.biopsycho.2017.05.003

Dennis-Tiwary, T. A., Egan, L. J., Babkirk, S., and Denefrio, S. (2016). For whom the bell tolls: neurocognitive individual differences in the acute stress-reduction effects of an attention bias modification game for anxiety. Behav. Res. Ther. 77, 105-117. doi: 10.1016/j.brat.2015.12.008

Dennis-Tiwary, T. A., Roy, A. K., Denefrio, S., and Myruski, S. (2019). Heterogeneity of the anxiety-related attention bias: a review and working model for future research. Clin. Psychol. Sci. 7, 879-899. doi: $10.1177 / 2167702619838474$

Denver, J. W., Reed, S. F., and Porges, S. W. (2007). Methodological issues in the quantification of respiratory sinus arrhythmia. Biol. Psychol. 74, 286-294. doi: 10.1016/j.biopsycho.2005.09.005 
Derryberry, D., and Reed, M. A. (2002). Anxiety-related attentional biases and their regulation by attentional control. J. Abnorm. Psychol. 111:225. doi: 10.1037/0021-843X.111.2.225

Dobbs, B., Pawlak, N., Biagioni, M., Agarwal, S., Shaw, M., Pilloni, G., et al. (2018). Generalizing remotely supervised transcranial direct current stimulation (tDCS): feasibility and benefit in Parkinson's disease. J. Neuroeng. Rehabil. 15:114. doi: 10.1186/s12984-018-0457-9

Egan, L. J., and Dennis-Tiwary, T. A. (2018). Dynamic measures of anxietyrelated threat bias: links to stress reactivity. Motivat. Emot. 42, 546-554. doi: 10.1007/s11031-018-9674-6

Eldar, S., Ricon, T., and Bar-Haim, Y. (2008). Plasticity in attention: implications for stress response in children. Behav. Res. Ther. 46, 450-461. doi: 10.1016/j.brat.2008.01.012

Elmasry, J., Loo, C., and Martin, D. (2015). A systematic review of transcranial electrical stimulation combined with cognitive training. Restor. Neurol. Neurosci. 33, 263-278. doi: 10.3233/RNN-140473

Emmelkamp, P. M. (2012). Attention bias modification: the Emperor's new suit? BMC Med. 10:63. doi: 10.1186/1741-7015-10-63

Fodor, L. A., Georgescu, R., Cuijpers, P., Szamoskozi, S., David, D., Furukawa, T. A., et al. (2020). Efficacy of cognitive bias modification interventions in anxiety and depressive disorders: a systematic review and network meta-analysis. Lancet Psychiatry 7, 506-514. doi: 10.1016/S2215-0366(20)30130-9

Fritsch, B., Reis, J., Martinowich, K., Schambra, H. M., Ji, Y., Cohen, L. G., et al. (2010). Direct current stimulation promotes BDNF-dependent synaptic plasticity: potential implications for motor learning. Neuron 66, 198-204. doi: 10.1016/j.neuron.2010.03.035

Gandiga, P. C., Hummel, F. C., and Cohen, L. G. (2006). Transcranial DC stimulation (tDCS): a tool for double-blind sham-controlled clinical studies in brain stimulation. Clin. Neurophysiol. 117, 845-850. doi: 10.1016/j.clinph.2005.12.003

Guleyupoglu, B., Schestatsky, P., Edwards, D., Fregni, F., and Bikson, M. (2013). Classification of methods in transcranial electrical stimulation (tES) and evolving strategy from historical approaches to contemporary innovations. J. Neurosci. Methods 219, 297-311. doi: 10.1016/j.jneumeth.2013.07.016

Hakamata, Y., Lissek, S., Bar-Haim, Y., Britton, J. C., Fox, N. A., Leibenluft, E., et al. (2010). Attention bias modification treatment: a meta-analysis toward the establishment of novel treatment for anxiety. Biol. Psychiatry 68, 982-990. doi: 10.1016/j.biopsych.2010.07.021

Heeren, A., Baeken, C., Vanderhasselt, M.-A., Philippot, P., and De Raedt, R. (2015). Impact of anodal and cathodal transcranial direct current stimulation over the left dorsolateral prefrontal cortex during attention bias modification: an eye-tracking study. PLOS ONE 10:0124182. doi: 10.1371/journal.pone.0124182

Heeren, A., De Raedt, R., Koster, E. H., and Philippot, P. (2013). The (neuro) cognitive mechanisms behind attention bias modification in anxiety: proposals based on theoretical accounts of attentional bias. Front. Human Neurosci. 7:119. doi: 10.3389/fnhum.2013.00119

Henry, J. D., and Crawford, J. R. (2005). The short-form version of the Depression Anxiety Stress Scales (DASS-21): construct validity and normative data in a large non-clinical sample. Br. J. Clin. Psychol. 44, 227-239. doi: 10.1348/014466505X29657

Hill, A. T., Rogasch, N. C., Fitzgerald, P. B., and Hoy, K. E. (2018). Effects of single versus dual-site High-Definition transcranial direct current stimulation (HD-tDCS) on cortical reactivity and working memory performance in healthy subjects. Brain Stimul. 11, 1033-1043. doi: 10.1016/j.brs.2018.06.005

Jarczok, M. N., Jarczok, M., Mauss, D., Koenig, J., Li, J., Herr, R. M., et al. (2013). Autonomic nervous system activity and workplace stressors-a systematic review. Neurosci. Biobehav. Rev. 37, 1810-1823. doi: 10.1016/j.neubiorev.2013.07.004

Kappenman, E. S., Farrens, J. L., Luck, S. J., and Proudfit, G. H. (2014). Behavioral and ERP measures of attentional bias to threat in the dot-probe task: poor reliability and lack of correlation with anxiety. Front. Psychol. 5:1368. doi: $10.3389 /$ fpsyg.2014.01368

Kasschau, M., Reisner, J., Sherman, K., Bikson, M., Datta, A., and Charvet, L. E. (2016). Transcranial direct current stimulation is feasible for remotely supervised home delivery in multiple sclerosis. Neuromodulation 19, 824-831. doi: 10.1111/ner.12430
Kazdin, A. E., and Blase, S. L. (2011). Interventions and models of their delivery to reduce the burden of mental illness: reply to commentaries. Perspect. Psychol. Sci. 6, 507-510. doi: 10.1177/1745691611418241

Kazdin, A. E., and Rabbitt, S. M. (2013). Novel models for delivering mental health services and reducing the burdens of mental illness. Clin. Psychol. Sci. 1, 170-191. doi: 10.1177/2167702612463566

Kessler, R. C., Berglund, P., Demler, O., Jin, R., Merikangas, K. R., and Walters, E. E. (2005). Lifetime prevalence and age-of-onset distributions of DSMIV disorders in the National Comorbidity Survey Replication. Arch. Gen. Psychiatry 62, 593-602. doi: 10.1001/archpsyc.62.6.593

Kronberg, G., Bridi, M., Abel, T., Bikson, M., and Parra, L. C. (2017). Direct current stimulation modulates LTP and LTD: activity dependence and dendritic effects. Brain Stimul. 10, 51-58. doi: 10.1016/j.brs.2016.10.001

Kronberg, G., Rahman, A., Sharma, M., Bikson, M., and Parra, L. C. (2020). Direct current stimulation boosts Hebbian plasticity in vitro. Brain Stimul. 13, 287-301. doi: 10.1016/j.brs.2019.10.014

Kruijt, A.-W., Parsons, S., and Fox, E. (2019). A meta-analysis of bias at baseline in RCTs of attention bias modification: no evidence for dot-probe bias towards threat in clinical anxiety and PTSD. J. Abnorm. Psychol. 128:563. doi: $10.1037 / \mathrm{abn} 0000406$

MacLeod, C., Mathews, A., and Tata, P. (1986). Attentional bias in emotional disorders. J. Abnorm. Psychol. 95:15. doi: 10.1037/0021-843X.95.1.15

MacLeod, C., Rutherford, E., Campbell, L., Ebsworthy, G., and Holker, L. (2002). Selective attention and emotional vulnerability: assessing the causal basis of their association through the experimental manipulation of attentional bias. J. Abnorm. Psychol. 111:107. doi: 10.1037/0021-843X.111.1.107

Martínez-Pérez, V., Castillo, A., Sánchez-Pérez, N., Vivas, A. B., Campoy, G., and Fuentes, L. J. (2019). Time course of the inhibitory tagging effect in ongoing emotional processing. A HD-tDCS study. Neuropsychologia 135:107242. doi: 10.1016/j.neuropsychologia.2019.107242

Mathews, A., and Mackintosh, B. (1998). A cognitive model of selective processing in anxiety. Cognit. Ther. Res. 22, 539-560. doi: 10.1023/A:1018738019346

Mathews, A., and MacLeod, C. (2002). Induced processing biases have causal effects on anxiety. Cogn. Emot. 16, 331-354. doi: 10.1080/02699930143000518

Matsumoto, D., and Ekman, P. (1989). American-Japanese cultural differences in intensity ratings of facial expressions of emotion. Motiv. Emot. 13, 143-157. doi: 10.1007/BF00992959

Miller, M. A., and Rahe, R. H. (1997). Life changes scaling for the 1990s. J. Psychosom. Res. 43, 279-292. doi: 10.1016/S0022-3999(97)00118-9

Mogoaşe, C., David, D., and Koster, E. H. (2014). Clinical efficacy of attentional bias modification procedures: an updated meta-analysis. J. Clin. Psychol. 70, 1133-1157. doi: 10.1002/jclp.22081

Molloy, A., and Anderson, P. L. (2020). Evaluating the reliability of attention bias and attention bias variability measures in the dot-probe task among people with social anxiety disorder. Psychol. Assess. 32:883. doi: 10.1037/pas0000912

Myruski, S., Bonanno, G. A., Gulyayeva, O., Egan, L. J., and Dennis-Tiwary, T. A. (2017). Neurocognitive assessment of emotional context sensitivity. Cogn. Affect. Behav. Neurosci. 17, 1058-1071. doi: 10.3758/s13415-017-0533-9

Nikolin, S., Loo, C. K., Bai, S., Dokos, S., and Martin, D. M. (2015). Focalised stimulation using high definition transcranial direct current stimulation (HDtDCS) to investigate declarative verbal learning and memory functioning. Neuroimage 117, 11-19. doi: 10.1016/j.neuroimage.2015.05.019

Nitsche, M. A., Cohen, L. G., Wassermann, E. M., Priori, A., Lang, N., Antal, A., et al. (2008). Transcranial direct current stimulation: state of the art 2008. Brain Stimul. 1, 206-223. doi: 10.1016/j.brs.2008.06.004

O'Shea, J., Boudrias, M.-H., Stagg, C. J., Bachtiar, V., Kischka, U., Blicher, J. U., et al. (2014). Predicting behavioural response to TDCS in chronic motor stroke. Neuroimage 85, 924-933. doi: 10.1016/j.neuroimage.2013.05.096

Pergamin-Hight, L., Pine, D. S., Fox, N. A., and Bar-Haim, Y. (2016). Attention bias modification for youth with social anxiety disorder. J. Child Psychol. Psychiat. 57, 1317-1325. doi: 10.1111/jcpp.12599

Porges, S. W. (1985). Method and Apparatus for Evaluating Rhythmic Oscillations in Aperiodic Physiological Response Systems. U.S. Patent No 4,510,944. Washington, DC: U.S. Patent and Trademark Office.

Price, R. B., Cummings, L., Gilchrist, D., Graur, S., Banihashemi, L., Kuo, S. S., et al. (2018). Towards personalized, brain-based behavioral intervention for transdiagnostic anxiety: transient neural responses to negative images predict 
outcomes following a targeted computer-based intervention. J. Consult. Clin. Psychol. 86:1031. doi: 10.1037/ccp0000309

Radman, T., Ramos, R. L., Brumberg, J. C., and Bikson, M. (2009). Role of cortical cell type and morphology in subthreshold and suprathreshold uniform electric field stimulation in vitro. Brain Stimul. 2, 215-228. doi: 10.1016/j.brs.2009.03.007

Rodebaugh, T. L., Scullin, R. B., Langer, J. K., Dixon, D. J., Huppert, J. D., Bernstein, A., et al. (2016). Unreliability as a threat to understanding psychopathology: the cautionary tale of attentional bias. J. Abnorm. Psychol. 125:840. doi: 10.1037/abn0000184

Rotheram-Borus, M. J., Swendeman, D., and Chorpita, B. F. (2012). Disruptive innovations for designing and diffusing evidence-based interventions. Am. Psychol. 67:463. doi: 10.1037/a0028180

Schmukle, S. C. (2005). Unreliability of the dot probe task. Eur. J. Person. 19, 595-605. doi: 10.1002/per.554

Schneider, W., Eschman, A., and Zuccolotto, A. (2002). E-Prime: User's Guide. Pittsburgh, PA: Psychology Software Incorporated.

Seibt, O., Brunoni, A. R., Huang, Y., and Bikson, M. (2015). The pursuit of DLPFC: non-neuronavigated methods to target the left dorsolateral pre-frontal cortex with symmetric bicephalic transcranial direct current stimulation (tDCS). Brain Stimul. 8, 590-602. doi: 10.1016/j.brs.2015.01.401

Shahbabaie, A., Hatami, J., Farhoudian, A., Ekhtiari, H., Khatibi, A., and Nitsche, M. A. (2018). Optimizing electrode montages of transcranial direct current stimulation for attentional bias modification in early abstinent methamphetamine users. Front. Pharmacol. 9:907. doi: 10.3389/fphar.2018.00907

Shaw, M. T., Kasschau, M., Dobbs, B., Pawlak, N., Pau, W., Sherman, K., et al. (2017). Remotely supervised transcranial direct current stimulation: an update on safety and tolerability. JoVE 128:e56211. doi: 10.3791/56211

Shen, B., Yin, Y., Wang, J., Zhou, X., McClure, S. M., and Li, J. (2016). Highdefinition tDCS alters impulsivity in a baseline-dependent manner. Neuroimage 143, 343-352. doi: 10.1016/j.neuroimage.2016.09.006

Spielberger, C. D. (1983). Manual for the State-Trait Anxiety Inventory STAI (Form Y)(“Self-Evaluation Questionnaire"). Palo Alto, CA:Consulting Psychologists Press. doi: 10.1037/t06496-000

Sprunger, J. G. (2018). Randomized Controlled Trial of an Attention-based Intervention for Alcohol-Facilitated Intimate Partner Aggression. West Lafayette, IN: Purdue University,

Stein, D. J., Medeiros, L. F., Caumo, W., and Torres, I. L. (2020). Transcranial direct current stimulation in patients with anxiety: current perspectives. Neuropsychiatr. Dis. Treat. 16:161. doi: 10.2147/NDT.S195840
Tottenham, N., Tanaka, J. W., Leon, A. C., McCarry, T., Nurse, M., Hare, T. A., et al. (2009). The NimStim set of facial expressions: judgments from untrained research participants. Psychiatry Res. 168, 242-249. doi: $10.1016 /$ j.psychres.2008.05.006

Valerio, J. (2004). An FMRI study of cardiovascular reactivity to mental stress. Faculty of Graduate Studies, University of Western Ontario, London, ON, Canada,

Waechter, S., and Stolz, J. A. (2015). Trait anxiety, state anxiety, and attentional bias to threat: assessing the psychometric properties of response time measures. Cognit. Ther. Res. 39, 441-458. doi: 10.1007/s10608-015-9670-Z

Wald, I., Bitton, S., Levi, O., Zusmanovich, S., Fruchter, E., Ginat, K., et al. (2017). Acute delivery of attention bias modification training (ABMT) moderates the association between combat exposure and posttraumatic symptoms: a feasibility study. Biol. Psychol. 122, 93-97. doi: 10.1016/j.biopsycho.2016.01.005

Weinberg, A., Venables, N. C., Proudfit, G. H., and Patrick, C. J. (2015). Heritability of the neural response to emotional pictures: evidence from ERPs in an adult twin sample. Soc. Cogn. Affect. Neurosci. 10, 424-434. doi: 10.1093/scan/nsu059

Woods, A. J., Antal, A., Bikson, M., Boggio, P. S., Brunoni, A. R., Celnik, P., et al. (2016). A technical guide to tDCS, and related non-invasive brain stimulation tools. Clin. Neurophysiol. 127, 1031-1048. doi: 10.1016/j.clinph.2015. 11.012

Conflict of Interest: TD-T has equity in Wise Therapeutics Inc, which owns Personal Zen, and is on the advisory board of Lil Space Inc. TD-T is an inventor, with IP under patent review, on a digital therapeutics system and cognitive training method related to Personal Zen. The City University of New York (CUNY) has IP on neurostimulation system and methods with author MB as inventors. MB has equity in Soterix Medical Inc and is a consultant for GSK, Halo, and $\mathrm{X}$.

The remaining authors declare that the research was conducted in the absence of any commercial or financial relationships that could be construed as a potential conflict of interest.

Copyright $\odot 2021$ Myruski, Cho, Bikson and Dennis-Tiwary. This is an open-access article distributed under the terms of the Creative Commons Attribution License (CC $B Y)$. The use, distribution or reproduction in other forums is permitted, provided the original author(s) and the copyright owner(s) are credited and that the original publication in this journal is cited, in accordance with accepted academic practice. No use, distribution or reproduction is permitted which does not comply with these terms. 